\title{
Double-Stranded RNA Binding Domain (dsRBD) of PKR Shows Variable Dynamics in the Presence of Bacteriophage Pf1: An NMR Insight and its Possible Implications
}

\author{
Geetika P. Agarwal and Ravi P. Barnwal ${ }^{*, \#}$ \\ Department of Chemical Sciences, Tata Institute of Fundamental Research (TIFR), Homi Bhabha Road, Colaba, \\ Mumbai-400005, India
}

\begin{abstract}
The human Protein Kinase R (PKR) is one of the important and critical components of the innate immune response against viral infection. It regulates distinct cellular functions and controls the fate of an RNA molecule in the cell. PKR dephosphorylation is characterised by inhibitory interactions between the kinase domain and the RNA binding domains (RBDs), but the complete structural details and dynamics of the latent state and its activation are not yet clear. Several studies on the activation of double stranded RNA binding domain (dsRBD) of Protein Kinase R (dsRBD-PKR) have been attempted during the last few decades. In order to further investigate on its activation mechanism, we have analysed the backbone $\left[{ }^{1} \mathrm{H}^{15} \mathrm{~N}\right]$-dynamics and chemical shift perturbation studies of dsRBD-PKR in the presence of bacteriophage Pf1. Pf1 is known to orient the protein in the presence of external magnetic field and, can be further used to get useful information about the protein properties such as the different dynamic behaviour in multidomain proteins like dsRBD-PKR. These NMR based results improve our basic understanding of the domain flexibilities of dsRBD-PKR where some secondary structural regions of dsRBM1 (dsRNA binding motif 1) show a different behaviour than the one from dsRBM2 (dsRNA binding motif 2) these observations further lead to a better understanding of PKR mechanism where dsRBM1 has more affinity towards RNA as compared to dsRBM2.
\end{abstract}

Keywords: PKR, protein kinase R, dsRBD, double stranded RNA binding domain, bacteriophage Pf1, dynamics, HSQC, heteronuclear single quantum coherence.

\section{INTRODUCTION}

The double-stranded RNA binding domain (dsRBD) of PKR, which was first identified in the early 1990s, is present in more than 100 proteins from different organisms ranging from Escherichia coli to humans. The dsRBDs are $\sim 70$ amino acid residues long and can only bind to a double stranded RNA (dsRNA). Recent studies suggest that it sometimes bind to a single-stranded RNA (ssRNA) or to a DNA $[1,2]$. These dsRBDs are classified into two distinct types based on their sequence comparison: Type-A dsRBDs and Type-B dsRBDs. Type-A dsRBDs are conserved over the entire length of a defined consensus sequence, whereas Type-B dsRBDs fit the consensus only at the carboxy terminal [2]. In vitro RNA-binding experiments show that only type-A dsRBDs bind to dsRNA, while type-B domains fail to bind dsRNA by themselves. However, the presence of multiple domains in tandem arrangement enhances their ability to bind dsRNA dramatically [3, 4]. Eukaryotic dsRNA binding proteins (DRBPs) may contain up to five dsRBDs, while other DRBPs coming from viruses usually contain only one.

\footnotetext{
*Address correspondence to this author at the Department of Chemical Sciences, Tata Institute of Fundamental Research (TIFR), Colaba, Mumbai400005, India; Tel: +91 222278 2271; Fax: +91 222280 4610;

E-mail: barnwal@tifr.res.in,barnwal@u.washington.edu

"Present Address: Department of Chemistry, University of Washington, Seattle- 98195, USA
}

The functions of DRBPs $[5,6]$ have been found to include RNA localisation, RNA editing, translational repression and most recently, post-translational gene silencing. It has been shown that proteins with multiple dsRBDs increase their functional diversity [5-7]. This has increased the interest of research in the field of DRBPs. Its importance has also been emphasised in brain tissues in patients with Alzheimer's, Parkinson's and Huntington's diseases [8].

The human Protein Kinase $R$ (PKR) is one of the most important antiviral proteins induced by interferons. Interferons are proteins secreted by the cellular defence system during viral infections. This protein is synthesised upon binding to dsRNA at latent state and undergoes autophosphorylation at various Ser and $\mathrm{Thr}$ residues, resulting in its activation. In addition to this, PKR is involved in different kinds of cellular signalling pathways.

Human PKR is a $68 \mathrm{kDa}$ long protein containing two domains, namely $\mathrm{N}$-terminal dsRNA-binding domain and the C-terminal catalytic or kinase domain. The N-terminal regulatory domain has two dsRNA binding motifs (the Nand the C-terminal motifs, generally abbreviated as dsRBM1 and dsRBM2, respectively). Each of these motifs is 70 amino acid residues long and is connected with a 20-22 residue long flexible linker. Despite extensive biochemical and genetic studies, the exact mechanism of the activation of PKR following dsRNA binding is not yet clearly understood. Though both dsRBMs of PKR are involved in dsRNA binding and PKR activation, they are functionally nonequivalent, with dsRBM1 exhibiting much higher dsRNA 
a

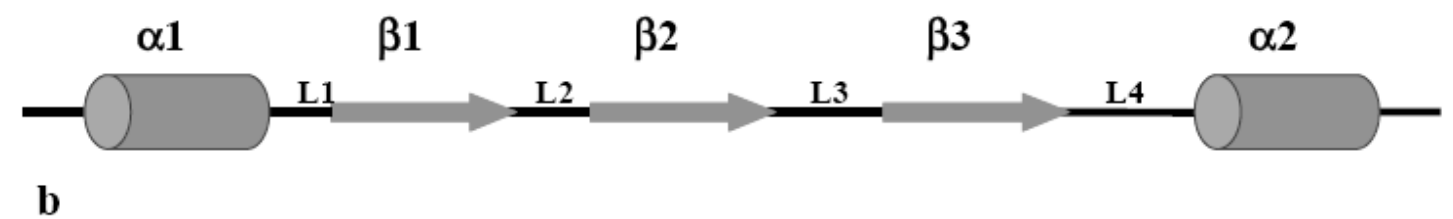

GSHMEMAGDLSAGF

FMEELNTYRQKQGVVLKYQELPNSGPPHDRRFTFQVIIDGREFPEGEGRSKKEAKNAAAKLAVEIL dSRBM1 NKEKKAVSPLLLTTTNSSEGLSMGN

YIGLINRIAQKKRLTVNYEQCASGVHGPEGFHYKCKMGQKEYSIGTGSTKQEAKQLAAKLAYLQI dSRBM2 LSEETGSGC

Region 1

Region 2

Region 3

Fig. (1). (a) Cartoon map defining primary and secondary structures for dsRBM1 and dsRBM2 of PKR. (b) Amino-acid sequence for dsRBD of PKR. Three regions shown are involved in dsRNA binding. Residues directly involved in interacting with dsRNA are coloured in red. Lines followed by the "dsRBM1" and "dsRBM2" represent residues involved in the respective motifs.

binding activity than dsRBM2 [4, 9-11]. Structural studies carried out using NMR as well as X-ray crystallography reveal the same kind of tertiary fold, $\alpha-\beta-\beta-\beta-\alpha$, for both the motifs (Fig. 1). The 3D-structure of dsRBD-PKR solved by NMR [12] further supports this kind of tertiary fold for both the motifs. Surprisingly, these domains exhibit specific and distinct functions, which are quite different from one another. Structure based understanding of such differential dsRNA binding has been limited because both motifs adopt similar folds with highly conserved RNA binding residues. These kinds of special behaviour and differences could be due to the protein flexibility and internal dynamics of the individual amino acid residues situated in the specific motifs, as described earlier [13, 14].

In this backdrop, we attempted to get information about PKR activation based on the backbone dynamics of these two motifs of dsRBD-PKR in the presence of bacteriophage Pf1, which is a medium to reorient the biomolecules in the presence of steric or magnetic effect $[15,16]$. The Pf1 consists of a single stranded circular DNA genome which is packaged at 1:1 nucleotide: coat-protein ratio. This packaging forms a rod of $\sim 60 \AA$ in diameter and $\sim 20,000 \AA$ in length, which orients with its long axis parallel to the external magnetic field. Bacteriophage Pf1 has been preferred over other media for alignment because of the following advantages: (a) It is highly stable in different experimental conditions $(\mathrm{pH}$ varying from 6.5-8.0 and different salt conditions), (b) unlike the bicelles medium, the ordered phase exists over a wide range of temperature $\left(5-45^{\circ} \mathrm{C}\right),(\mathrm{c})$ the phage fully aligns over a wide range of phage concentrations $(1-50 \mathrm{mg} / \mathrm{ml})$. In addition, Pf1 [17-20] has negatively charged surface at physiological $\mathrm{pH}$ like RNA and DNA oligomer. We have used dsRBD-PKR in the presence of Pf1 to measure RDC (residue dipolar couplings) which will be published elsewhere. During the course of RDC measurement, we performed NMR dynamics to get information of dsRBD in the presence of Pf1 which seems to be quite interesting and promising up to certain extent. Though Pf1 is quite different from dsRNA, and dsRBD-PKR is quite specific to the dsRNA, these findings can be used as an addition to our earlier reported studies $[13,14]$ and in fact, increase our understanding of PKR activation where some secondary structural regions of dsRBM1 may anchor the RNA primarily, in a more efficient fashion than dsRBM2.

\section{RESULTS AND DISCUSSION}

\section{(a) NMR ${ }^{15} \mathrm{~N}$ - Relaxation Dynamics of dsRBD in the Presence of Bacteriophage Pf1}

The following NMR relaxation parameters were collected at two different magnetic field strengths (500 and $600 \mathrm{MHz}$ ) to calculate the motional properties of dsRBD containing the two dsRBMs and the central linker: (a) spinlattice relaxation time $\left(\mathrm{T}_{1}\right)$ (Fig. 2A); (b) spin-spin relaxation time $\left(\mathrm{T}_{2}\right) \quad$ (Fig. 2B); (c) steady-state ${ }^{1} \mathrm{H}^{15} \mathrm{~N}$ nuclear Overhauser effects (NOEs) (Fig. 2C). Based on the ${ }^{1} \mathrm{H} /{ }^{15} \mathrm{~N}$ backbone chemical shift assignments of dsRBD in Pf1, the relaxation data for 143 out of 179 residues present in dsRBD were used in the analysis. The remaining were prolines, residues showing spectral overlap, the one at the N-terminal end which broadened out due to chemical exchange, and the residues that did not show their signatures in HSQC spectrum. Here, the dsRBD sequence includes extra amino acid residues, which arise from the cloning, five at the $\mathrm{N}$ terminal end and four at the C-terminal end.

The average $T_{1}, T_{2}$ values and NOEs for residues with regular secondary structures in dsRBM1 and dsRBM2 are given in Table 1. As observed commonly in native proteins, the observed $T_{1}$ values are almost constant throughout the sequence and hence, can not be used directly to sense the motional properties of the polypeptide chain. The $T_{2}$ values are also uniform except for residues numbered $35,36,85-$ $106,140,141,142$, which show higher values of $T_{2}$. These higher values of $T_{2}$, particularly from the residues 85 to 106 region, originated from the flexibility of this amino-acid segment linking the two-domains. In the absence of conformationally exchanging motions, and provided that the extreme narrowing condition for fast internal motions $\left(\omega_{0} \tau_{\mathrm{c}}<<1\right.$, where $\omega_{0}$ is the Larmor frequency, and $\tau_{\mathrm{c}}$ is the correlation time for the internal motion of the $i^{\text {th }} \mathrm{NH}$ vector) is satisfied, the correlation frequencies for internal motions affect $T_{1}$ and $T_{2}$ to the same extent. Under these circumstances, the $T_{1} / T_{2}$ ratio depends only on the overall molecular tumbling correlation time, $\tau_{\mathrm{m}}\left(\tau_{\mathrm{c}}\right)[21,22]$. Thus, the $\mathrm{T}_{1} / \mathrm{T}_{2}$ 

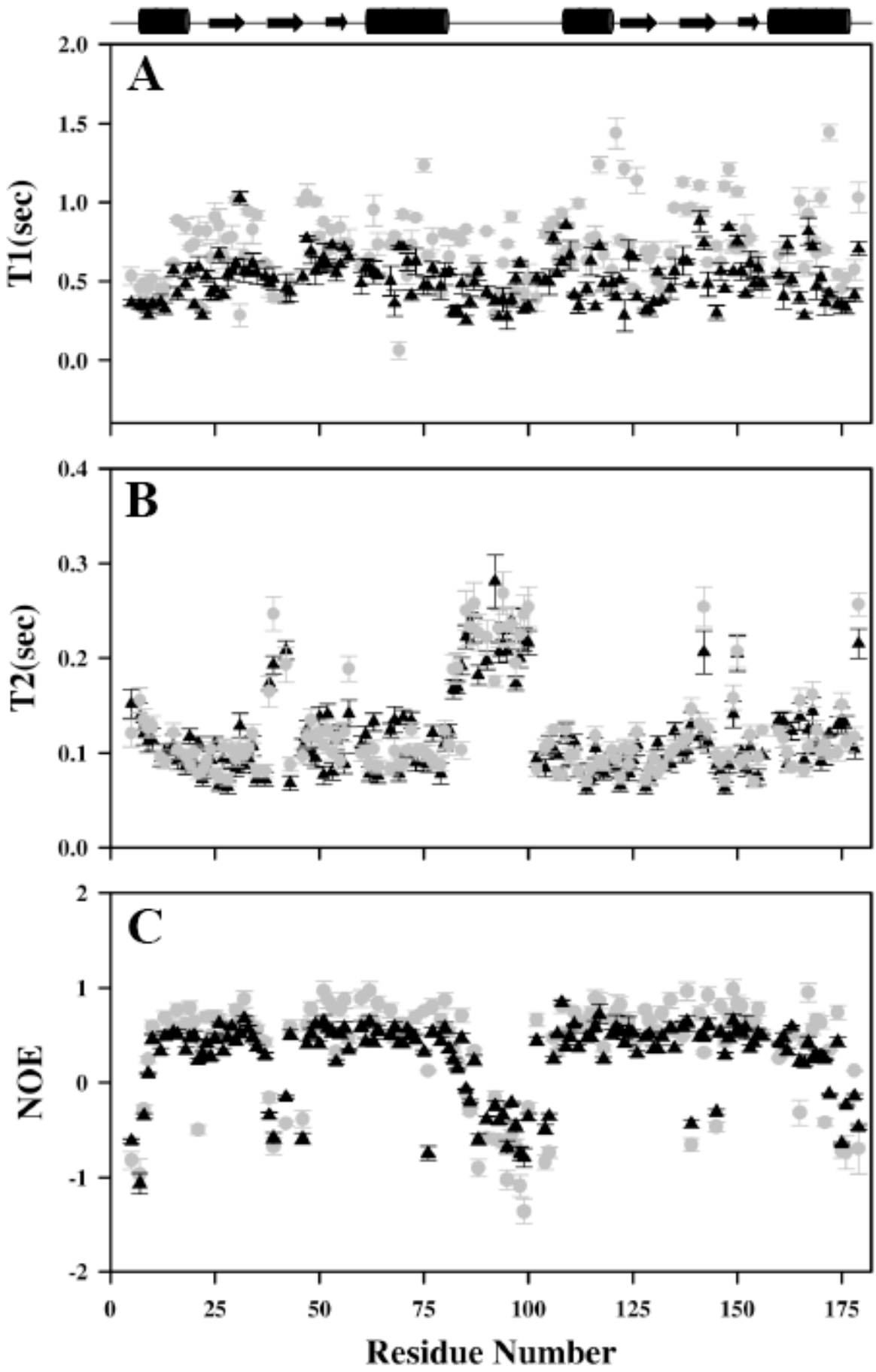

Fig. (2). ${ }^{15} \mathrm{~N}$ relaxation data at $11.7 \mathrm{~T}$ (black triangles) and $14.1 \mathrm{~T}$ (grey circles). A, Longitudinal relaxation times, T1 (1/R1). B, Transverse relaxation times, T2 $(1 / \mathrm{R} 2) \cdot \mathbf{C},\left[{ }^{1} \mathrm{H}_{-}{ }^{15} \mathrm{~N}\right] \mathrm{NOE}$ enhancements are defined as $\mathrm{I}_{\mathrm{sat}} / \mathrm{I}_{\mathrm{eq}}$ where $\mathrm{I}_{\mathrm{sat}}$ and $\mathrm{I}_{\mathrm{eq}}$ are the intensities of peak in the $2 \mathrm{D}$ experiments with and without proton saturation, respectively. Error bar of the T1 and T2 data denote curve-fitting uncertainties: errors in the $\left[{ }^{1} \mathrm{H}^{-15} \mathrm{~N}\right]$ NOEs are estimated from the signal/noise ratio of the spectra. Arrows $(\boldsymbol{\longrightarrow})$ denote $\beta$-sheets and cylinders $(\boldsymbol{\infty})$ denote $\alpha$-helices.

ratio provides a useful initial information/estimate of $\tau_{\mathrm{m}}$. Residues with large-amplitude internal motions in a timescale longer than a few hundreds of picoseconds, which can be identified by low NOE values (0.6), must be excluded from this calculation. By following the above procedures, the mean $\mathrm{T}_{1} / \mathrm{T}_{2}$ ratios were found to be $8.25 \pm 0.36$ at $600 \mathrm{MHz}$ and $5.95 \pm 0.26$ at $500 \mathrm{MHz}$. The ratio yielded the initial estimate of tm of $8.95 \pm 0.12$ and $9.75 \pm 0.18$ for $600 \mathrm{MHz}$ and
$500 \mathrm{MHz}$, respectively. Fig. (S1) shows the $T_{1} / \mathrm{T}_{2}$ ratio as a function of the amino acid sequence of dsRBD-PKR.

\section{(b) Overall Rotational Correlation Time, $\tau_{m}$ or $\tau_{c}$}

The overall rotational correlation times calculated for dsRBD-PKR in the presence of $5 \mathrm{mg}$ of Pf1 were $9.75 \pm 0.18$ and $8.95 \pm 0.12 \mathrm{~ns}$ for 500 and $600 \mathrm{MHz}$, respectively. This 
Table 1. Comparison of Backbone Dynamics Parameters for dsRBM1 and dsRBM2 of dsRBD-PKR in Presence of Bacteriophage Pf1

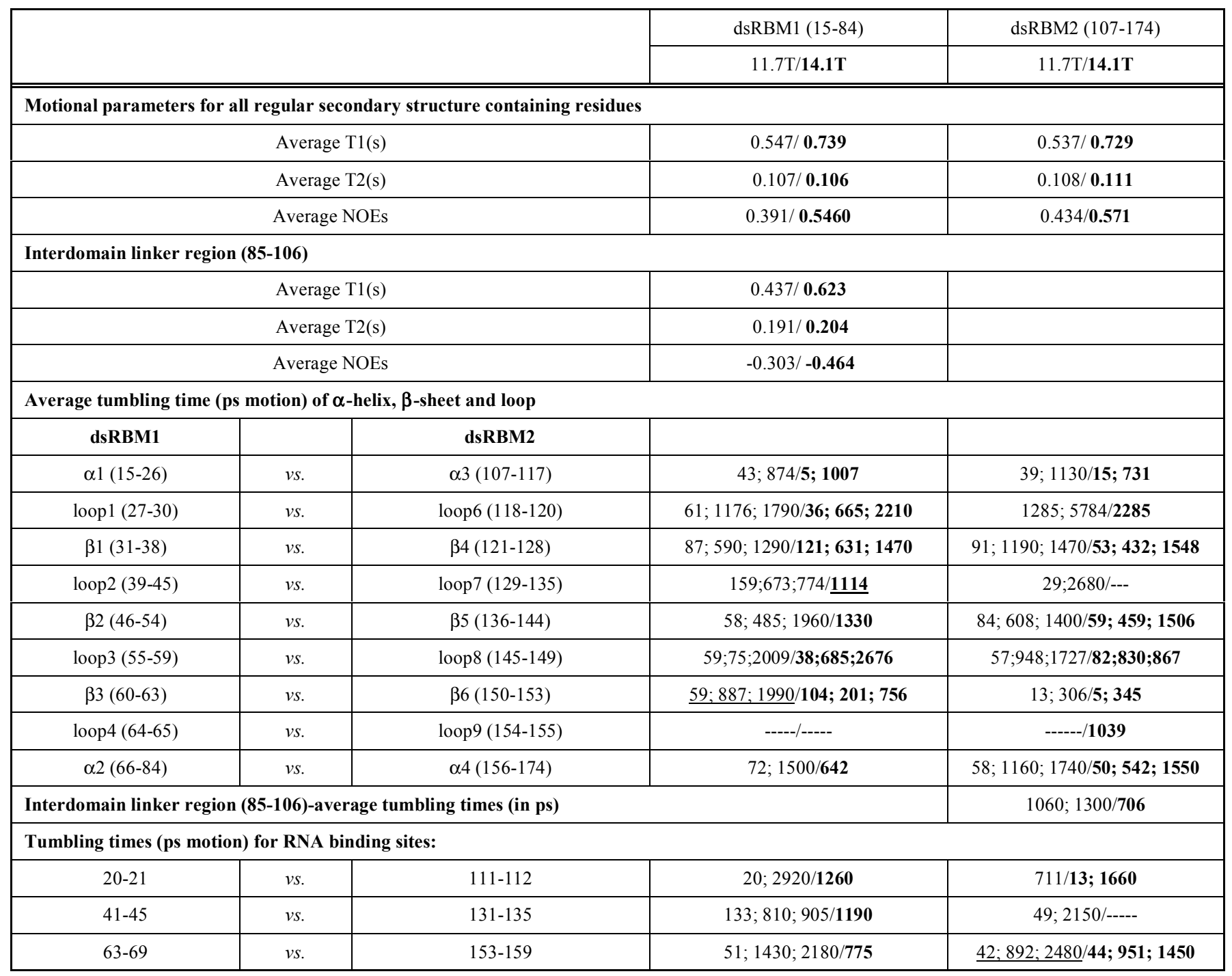

Bold ones are for $600 \mathrm{MHz}(14.1 \mathrm{~T})$. Underlined one has an additional value (not shown) in millisecond-microsecond regime.

value is slightly different than the overall correlation time, $9.53 \pm 0.03 \mathrm{~ns}$, reported in the case of free protein [14]. This marginal increase/lowering of overall correlation time could be due to the presence of Pf1 in dsRBD-PKR.

\section{(c) $\tau_{m}$ Determination from the Reduced Spectral Density Map}

The three spectral density parameters $J\left(\omega_{H}\right), J\left(\omega_{N}\right)$ and $J(0)$ were calculated as mentioned in materials and methods section (Fig. S2). Only a few residues showed distinctly higher values of $J(0)$, indicating that chemical exchange motions have significant contributions to the mobility of their NH vectors. A linear correlation between $J\left(\omega_{N, H}\right)$ and corresponding $J(0)$ values using the equation $\mathrm{J}\left(\omega_{\mathrm{N}, \mathrm{H}}\right)=\alpha$ $\mathrm{J}(0)+\beta$, has been proposed for the calculation of $\tau_{\mathrm{m}}[23]$. The $\alpha$ - and $\beta$-values from the plot of $J\left(\omega_{N}\right) v s . J(0)$ were found to be -0.0075 and $0.2763 \mathrm{~ns} / \mathrm{rad}$, respectively for $600 \mathrm{MHz}$ and -0.0153 and $0.3901 \mathrm{~ns} / \mathrm{rad}$, respectively for $500 \mathrm{MHz}$. These values were used to calculate the overall molecular tumbling correlation time $\left(\tau_{\mathrm{m}}\right)$ from the equation $[23,24]$ :

$$
2 \alpha \omega_{(\mathrm{H}, \mathrm{N})}{ }^{2} \tau_{\mathrm{m}}{ }^{3}+5 \beta \omega_{(\mathrm{H}, \mathrm{N})}{ }^{2} \tau_{\mathrm{m}}{ }^{2}+2(\alpha-1) \tau_{\mathrm{m}}+5 \beta=0
$$

The solution of the above cubic equation yielded three values $\left(\mathrm{x} 1, \mathrm{x} 2\right.$, and $\mathrm{x} 3$ ) of $\tau_{\mathrm{m}}$ (Table 2$)$. The values in millisecond, microsecond, sub-ns and picoseconds represent the chemical exchange/overall rotational tumbling time or internal motion depending on their amplitudes.

Table 2. Correlation Times Calculated at Different Magnetic Field

\begin{tabular}{|c|c|}
\hline Spectrometer Field & Correlation Times $(\mathbf{x} 1, \mathbf{x} 2$, and x3) \\
\hline \hline $11.7 \mathrm{~T}\left[\right.$ from $\mathrm{J}\left(\omega_{\mathrm{H}}\right)$ and $\left.\mathrm{J}(0)\right]$ & $10.0 \mathrm{~ns}, 158.1 \mathrm{ps}, 693.1 \mathrm{ps}$ \\
\hline $11.7 \mathrm{~T}\left[\right.$ from $\mathrm{J}\left(\omega_{\mathrm{N}}\right)$ and $\left.\mathrm{J}(0)\right]$ & $51.2 \mathrm{~ns}, 11.3 \mathrm{~ns}, 1100 \mathrm{ps}$ \\
\hline $14.1 \mathrm{~T}\left[\right.$ from $\mathrm{J}\left(\omega_{\mathrm{H}}\right)$ and $\left.\mathrm{J}(0)\right]$ & $8.2 \mathrm{~ns}, 163.3 \mathrm{ps}, 464.0 \mathrm{ps}$ \\
\hline $14.1 \mathrm{~T}\left[\right.$ from $\mathrm{J}\left(\omega_{\mathrm{N}}\right)$ and $\left.\mathrm{J}(0)\right]$ & $8.0 \mathrm{~ns}, 10.1 \mathrm{~ns}, 731 \mathrm{ps}$ \\
\hline
\end{tabular}




\section{(d) The $\mathbf{R}_{\mathrm{ex}}$ Term}

$J(0)$ may contain chemical exchange $\left(\mathrm{R}_{\mathrm{ex}}\right)$ contributions according to: $\mathrm{J}(0)=\mathrm{J}_{\text {int }}(0)+\lambda \mathrm{R}_{\mathrm{ex}}[23,25]$. In the absence of $\mathrm{R}_{\mathrm{ex}}$ contributions, $J(0)$ is equal to $\mathrm{J}_{\text {int }}(0)$ therefore, the obtained $J(0)$ values should be field independent. However, in the presence of $R_{\text {ex }}, J(0)$ is expected to increase with field strength, since $\mathrm{R}_{\mathrm{ex}} \propto \omega_{\mathrm{N}}{ }^{2}$ (Fig. S3).

$\mathrm{R}_{\mathrm{ex}}$-contributions at $600 \mathrm{MHz}$ as well as $500 \mathrm{MHz}$ were calculated based on the following equation,

$$
\begin{aligned}
\mathrm{R}_{\mathrm{ex}}, \omega \mathrm{N}=\omega_{\mathrm{N}}^{2}\left[\{\mathrm{~J}(0)\}_{14.1 \mathrm{~T}}-\{\mathrm{J}(0)\}_{11.7 \mathrm{~T}}\right] \\
\\
{\left[\lambda_{14.1 \mathrm{~T}} \omega_{\mathrm{N}, 14.1 \mathrm{~T}}^{2}-\lambda_{11.7 \mathrm{~T}} \omega_{\mathrm{N}, 11.7 \mathrm{~T}}^{2}\right]^{-1} }
\end{aligned}
$$

We emphasised that though the intrinsic errors of such estimation of $R_{e x}$ is always large [25], it can still be used as a good interpretation of the experimental data.

\section{(e) $\left[{ }^{1} \mathrm{H}-{ }^{15} \mathrm{~N}\right]-$ Backbone Dynamics and Internal Motions}

The backbone relaxation data analysis was done for 143 out of 179 backbone ${ }^{15} \mathrm{~N}$ nuclei of dsRBD of PKR. The dsRBM1 contained 70 residues, the dsRBM2 had 68 residues, the interdomain linker had 22 residues, the polypeptide stretch at the $\mathrm{N}$-terminal end (not part of dsRBM1) contained 14 residues, and C-terminal part of dsRBD (not part of dsRBM2) had 5 residues. However, we could only account for 58 residues coming from dsRBM1, 56 residues from dsRBM2, and 18 residues from interdomain linker in relaxation data analysis. The average $T_{1}, T_{2}$, NOE and internal motions for regular secondary structure as well as interdomain linker are given in Table 1. Further, all tumbling motions measured for RNA binding sites in ps are presented in Table 1. As seen from Fig. (2) and Table 1, average $T_{1}, T_{2}$ values for the residues with regular secondary structures for dsRBM1 and dsRBM2 are almost similar, whereas the average NOEs for dsRBM1 is smaller than dsRBM2 at both
500 and $600 \mathrm{MHz}$. It is evident from Fig. (2) that residues with rigid and regular secondary structures in the protein, either from dsRBM1 or from dsRBM2, showed smaller $T_{2}$ and higher NOE values than flexible loop residues. The interdomain linker (85-106) showed a relatively higher $\mathrm{T}_{2}$ and low (negative) NOE values (Fig. 3). These rigid and regular secondary structural components exhibit different varieties of internal motions (Table 1).

The internal motions for loops/turns for dsRBMs are given in Table 1. Loop2/loop 7, poorly defined in NMR structure [12] and the main part of dsRNA binding, showed less internal motions in the presence of bacteriophage Pf1, as seen in the original dynamics study (Nanduri et al. 2000). Similarly $\alpha 1, \alpha 3$, loop4 and loop9 exhibited less internal motions. This may be due to their close proximity to the dsRNA binding sites. Interestingly, all dsRNA binding partners coming from dsRBM1 had less internal motion than those present in dsRBM2. This could lead to a conclusion that all partners in dsRBM1 are more affected in presence of the bacteriophage Pf1 or $d s R N A$. This might add some more information about dsRBD-PKR activation where dsRBM1 may anchor to the bacteriophage Pf1/dsRNA primarily and then dsRBM2 comes into play. Since dsRBM2 also interacts with kinase domain, there is a possibility that it may interact at a later stage in the activation mechanism. In dsRBM1, there are mainly three RNA binding regions and from Table 1, it is evident that most of them have fewer motions as mentioned above. Since loop4 shows no internal motions in the presence of Pf1, it can be concluded that it may have got affected with Pf1 first. This suggests that loop3 may be involved in the interaction with dsRNA first, followed by loop2 and $\alpha 1$ approaching the dsRNA. These results were supported by $\mathrm{R}_{\mathrm{ex}}$-plot in the supplementary (Fig. S3), in which all RNA binding regions from dsRBM1 have distinct and different values than binding regions present in dsRBM2. In this $R_{e^{-}}-p l o t$, loop4 which comes from dsRBM1 had less $R_{\mathrm{ex}}$ values than its counterpart from dsRBM2 which further suggests the primary involvement of

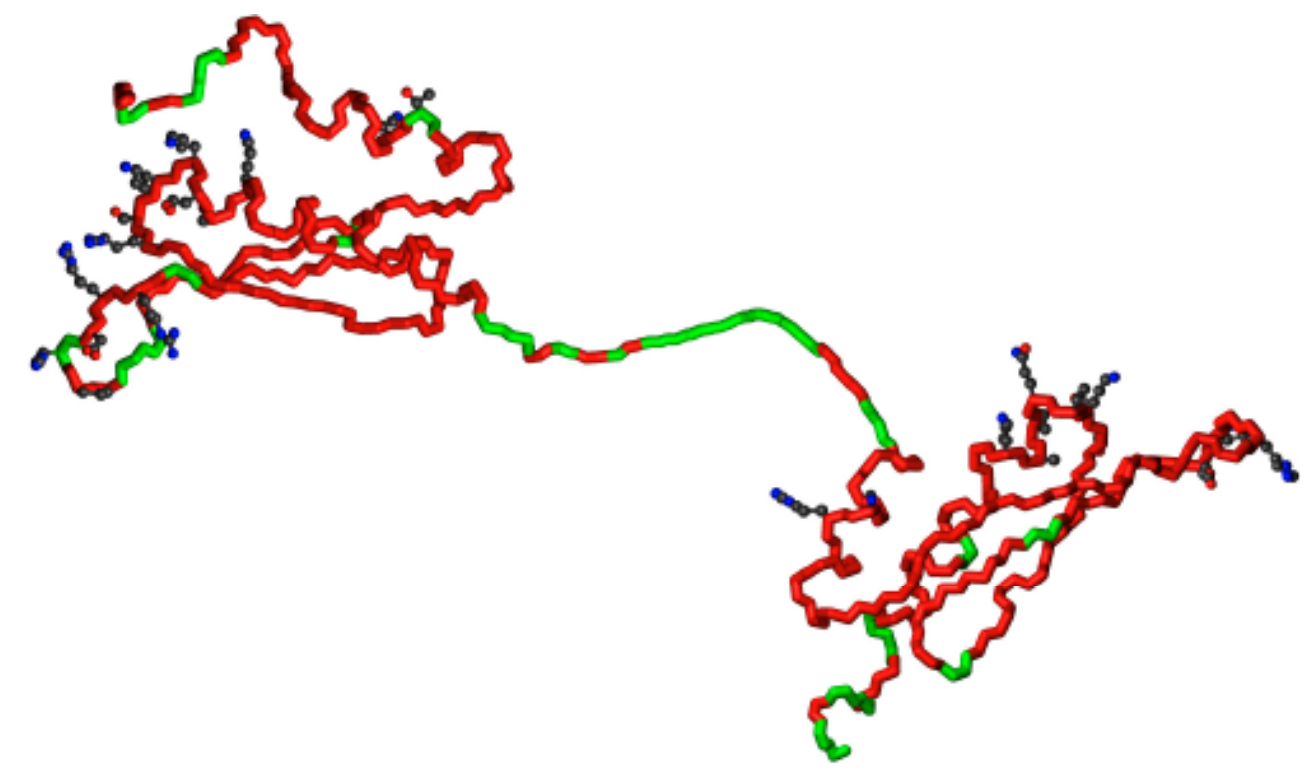

Fig. (3). The annotated picture for backbone residues in dsRBM1/dsRBM2 using MOLMOL [45] (www.mol.biol.ethz.ch/wuthrich/software/ molmol), showing high flexibility with green and low flexibility with red colour. Residues (RNA binding residues) which are majorly affected by bacteriophage pf1 are shown with side chain. (BMRB ID: 4110; PDB: 1QU6). 
loop4 in dsRNA binding followed by $\alpha 1$ and loop 2 so as to make it complete, followed by dsRBM 2 anchoring.

\section{(g) Chemical Shift Perturbation Studies, RNA Binding and Model for PKR Activation}

We used chemical shift perturbation for getting the information about dsRNA binding of dsRBD-PKR. In this case, we used ${ }^{1} \mathrm{H}$ and ${ }^{15} \mathrm{~N}$ chemical shift differences recorded in $\left[{ }^{1} \mathrm{H}^{15} \mathrm{~N}\right]-\mathrm{HSQC}$ of dsRBD in its free form (BMRB 4110) and complexed with bacteriophage Pf1. Here, all assignments for dsRBD-PKR in the presence of Pf1 were rechecked using multinuclear NMR experiments. The average chemical shift perturbation, $\Delta \delta$ ( $\mathrm{ppm})$, was determined according to the following equation, followed by three point averaging for the smoothening of data (Fig. 4).

$$
\Delta \delta(\mathrm{ppm})=\left[\left(\delta \mathrm{H}^{\mathrm{N}}\right)^{2}+(\delta \mathrm{N} / 10)^{2}\right]^{0.5}
$$

The chemical shift perturbation mapping of the dsRBD of PKR in the presence of bacteriophage Pf1 is shown in Fig. (4). Residues which exhibited significant changes in the presence of bacteriophage Pf1 are situated in the region marked by "grey colour bars". These grey coloured regions mainly include dsRNA binding region during PKR activation. A potentially interesting observation in the differences between the two dsRBMs of dsRBD-PKR is the residues 42/43 of loop2 (from dsRBM1) and residues 128/130 of loop 7 (from dsRBM2). These were shown to be the primary loops involved in dsRNA binding $[13,14]$. In both the dsRBMs, the residues with relatively significant chemical shift perturbations were mapped on the helix $\alpha 1$, loop2 $(\beta 1$ $\beta 2$ loop) and loop4 ( $\beta 3-\alpha 2$ loop and N-terminal of helix $\alpha 2)$. There are some other regions which showed slightly high perturbation, which can not be explained presently by us. This seems to be consistent with the previously reported structures of single dsRBD-dsRNA complexes [26-28]. In these structures, three regions of dsRBD of PKR interacted with the dsRNA. The first region, helix $\alpha 1$, interacted with the minor groove of the dsRNA and the successive major groove of the dsRNA was recognised by the second region, which is loop4 and loop2. Here, perturbation data show that both the dsRBMs of PKR are mildly affected with the bacteriophage Pf1, primarily in the helix $\alpha 1$, the loop2 $(\beta 1$ $\beta 2$ loop) and the loop4 ( $\beta 3-\alpha 2$ loop and N-terminal of helix $\alpha 2)$ of dsRBD of PKR (Figs. 3, 4). In contrast, loop2 and loop4 showed more chemical shift perturbations in the presence of bacteriophage Pfl and can be explained by Fig. (5). In this model, loop4 primarily interacted with dsRNA followed by other dsRNA binding regions becoming available to bind dsRNA. Following this, dsRBM2 tried to anchor for dsRNA binding but had a lesser preference. In loop2/loop7, there was always at least one positively charged residue, which interacted with the minor groove of dsRNA and is shown in Fig. (1). In addition to these three main dsRNA binding regions, the residues from the interdomain linker present in between the two dsRBMs also exhibited significant chemical perturbations (Fig. 4). Based on the earlier studies done for ssRBDs [29, 30], these chemical shift perturbations can be explained. The present significant perturbations of the interdomain linker might be due to the direct interaction with the dsRNA or conformational change of the linker or both the dsRNA and the interdomain linker. In either ways, our chemical shift perturbation data showed that the interdomain linker seems to be a good candidate for dsRNA binding recognition.

In summary, we have shown that both the dsRBMs in PKR show different backbone dynamics and different chemical shift perturbation in the presence of Pfl. Though these chemical shift perturbations are less and Pf1 is quite different from dsRNA, these results can be correlated well with dsRBD-PKR's differential dsRNA binding activities as shown in the model (Fig. 5). Though for the complete understanding of the dsRNA binding and involvement of the dsRBD secondary structural elements, one needs to observe these changes in presence of dsRNA, yet these studies give further understanding of RNA binding to the protein. We further comment that the interdomain linker may be involved in dsRNA binding recognition. These results propose a dynamically tuned dsRNA binding mechanism for PKR

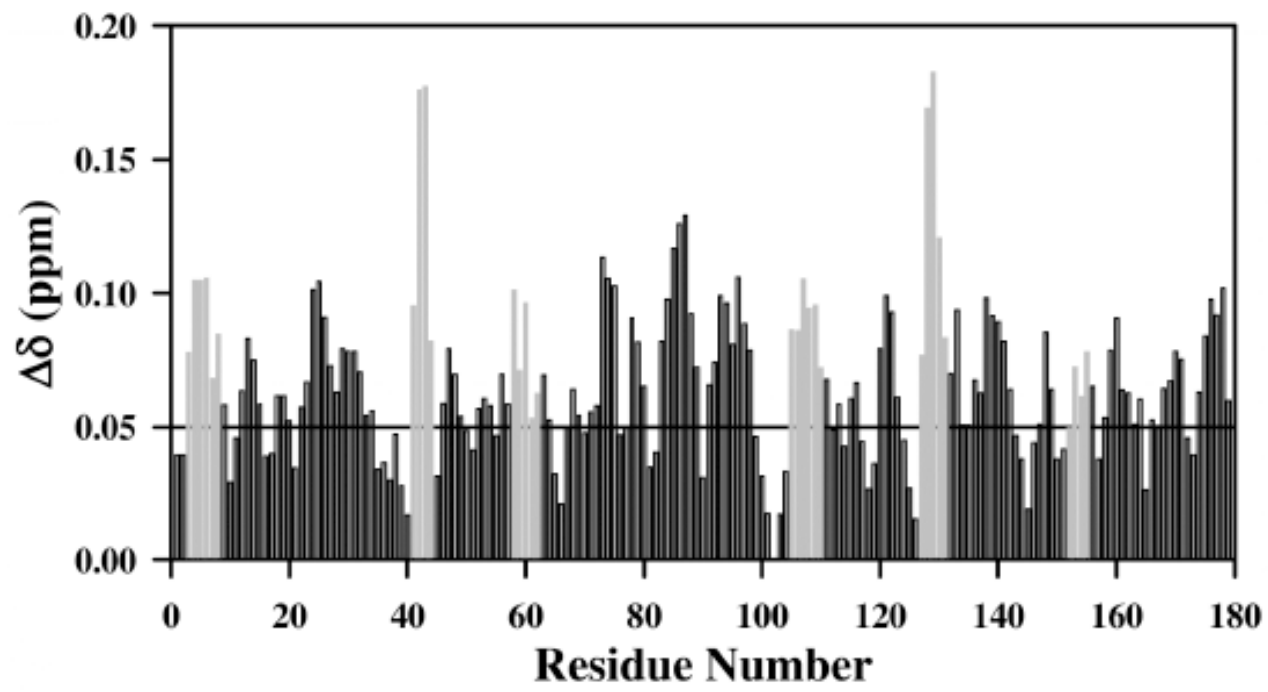

Fig. (4). The profile of chemical shift perturbations. Plots of the weighted averaged $\mathrm{H}$ and $\mathrm{N}$ chemical shift changes calculated with the function $\Delta \delta=\left[\left(\delta \mathrm{H}^{\mathrm{N}}\right)^{2}+(\delta \mathrm{N} / 10)^{2}\right]^{0.5}$. The most affected regions with bacteriophage Pf1 are shown in grey coloured filled bars. The horizontal line is drawn at $0.05 \mathrm{ppm}$ to discriminate the regions of major perturbations. 


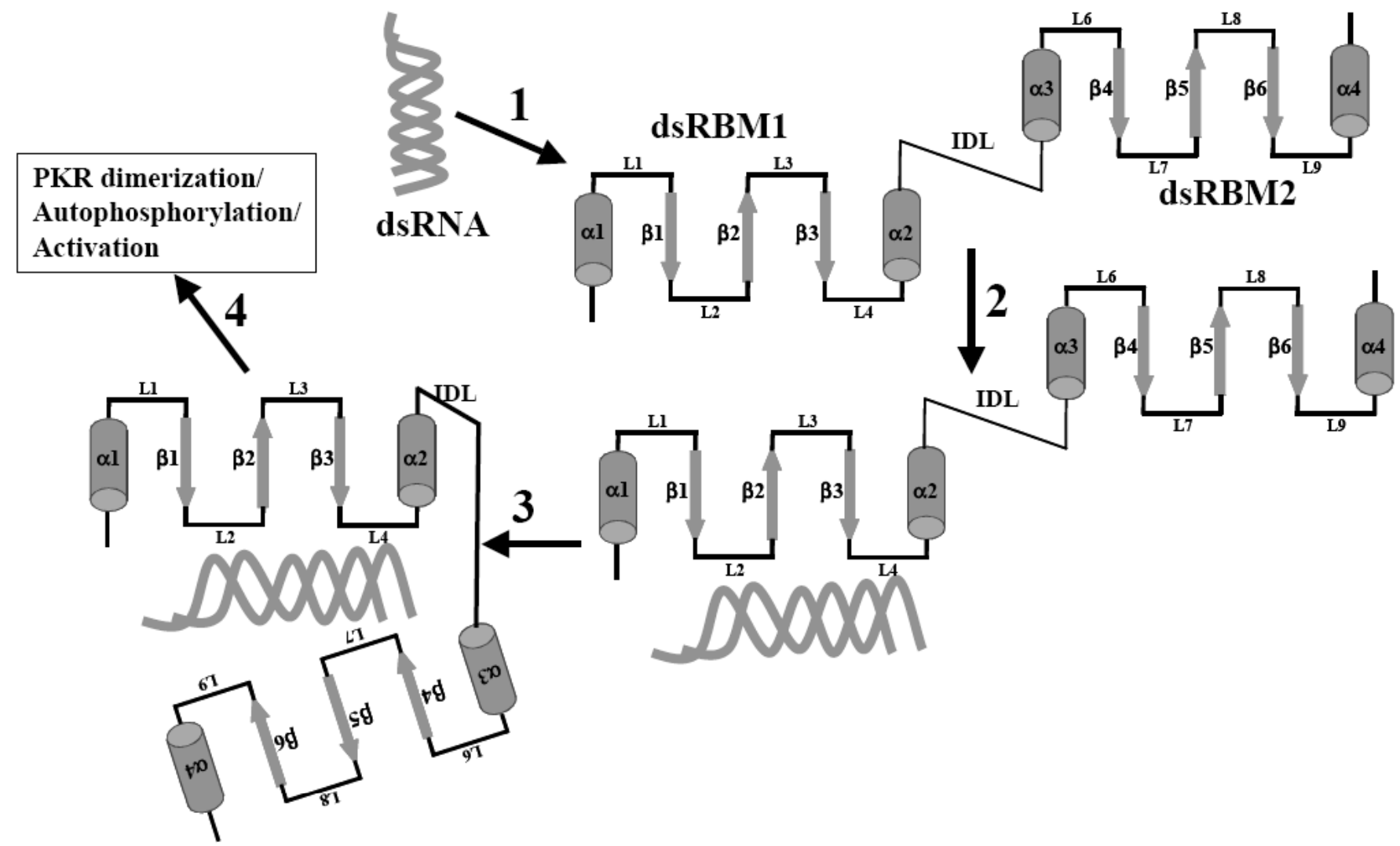

Fig. (5). Basic model for dsRNA-mediated PKR activation. Viral dsRNA is first anchored to the free dsRBM1, towards loop4 and loop2 followed by $\alpha 1$. This induces the cooperative binding to dsRBM2, which then gets exposed to bind to the kinase domain. This results in PKR dimerisation, authophosphorylation and activation. IDL denotes interdomain linker.

activation where loop4 and loop2 of dsRBM1 may anchor primarily to dsRNA and then $\alpha 1$ region for complete support. This might have induced the cooperative RNA binding to dsRBM2. These studies could lead to a mechanism about PKR activation as similar to the one mentioned in [14], where the involvement of specific secondary structure segments in the activation pathway is briefly explained.

\section{MATERIALS AND METHODS}

\section{Sample Preparation}

The ${ }^{15} \mathrm{~N}$-dsRBD-PKR sample was over-expressed and purified using the protocol reported earlier [31]. The monomeric state of dsRBD-PKR was confirmed using gelfiltration column. This observation is consistent with its overall correlation time $\left(\tau_{\mathrm{c}}=9.5 \mathrm{~ns}\right)$ [12]. The bacteriophage Pf1 was purchased from ASLA Biotech (http://www.aslabiotech.com). We used $5.0 \mathrm{mg} / \mathrm{ml}$ of bacteriophage Pf1 for the alignment, which was sufficient enough to orient the protein and study the protein dynamics. At higher concentrations of bacteriophage, the protein precipitates were observed.

\section{NMR Experiments and Data Analysis}

All the NMR experiments were recorded either on Varian Inova $600 \mathrm{MHz}$ or Bruker Avance $500 \mathrm{MHz}$ spectrometers at $25{ }^{\circ} \mathrm{C}$, each equipped with a triple-resonance probe head and a shielded z-gradient unit. Few GFT based experiments were used to assign the dsRBD-PKR in the presence of Pf1 [32-34].

${ }^{15} \mathrm{~N}$ relaxation parameters, $\mathrm{T}_{1}, \mathrm{~T}_{2}$ and ${ }^{1} \mathrm{H}-{ }^{15} \mathrm{~N}$ heteronuclear NOEs were obtained using the experiments described earlier [35]. $\mathrm{T}_{1}$ experiments were done with the following delays of $11.2,89.7 *, 190.6,203.7 *$, 437.2, $605.4 *, 807.2$ and $1076.3 \mathrm{~ms}$ at $600 \mathrm{MHz}$ spectrometer frequency, while the corresponding delays were $12.5,91.0 \%$, $192.0,304.0 *, 438.5,606.7 *, 808.7$ and $1077.6 \mathrm{~ms}$ at 500 $\mathrm{MHz}$. The $\mathrm{T}_{2}$ experiments were recorded with delay times of $16.7,33.4 *, 50.1,66.8 *, 83.5,100.5 *, 116.9$ and $133.6 \mathrm{~ms}$ at $600 \mathrm{MHz}$, while the corresponding delays were 16.5, 33.0*, $49.5,66.0 *, 82.5,99.0 *, 115.5$ and $132.0 \mathrm{~ms}$ at $500 \mathrm{MHz}$. The experiments with the asterisk $(*)$ were run twice to check the reproducibility of both the $T_{1}$ and $T_{2}$ measurements. Two NOE experiments were collected: one with a $3.0 \mathrm{~s}$ recycle delay followed by a $3.0 \mathrm{~s}$ pre-saturation period, and the other with a $6 \mathrm{~s}$ recycle delay without any pre-saturation at both the spectrometer frequencies, 600 and $500 \mathrm{MHz}$. Two dimensional $\left[{ }^{1} \mathrm{H}^{-15} \mathrm{~N}\right]$-HSQC spectra were recorded before and after the collection of the entire relaxation data to check the stability of the protein. No changes were found during the entire course of data collection, which took around $72 \mathrm{hrs}$ in total.

All the data were processed using Felix 97 software (Molecular Simulations Inc.). The spectra were typically apodized using a $60^{\circ}$-shifted sine square bell window functions before zero-filling in both the dimensions, followed by Fourier transformation. 


\section{Determination of ${ }^{15} \mathrm{~N}$-Relaxation Parameters $\left(\mathbf{R}_{1}, \mathbf{R}_{\mathbf{2}}\right.$, and NOE)}

$\mathrm{R}_{1}\left(\mathrm{~T}_{1}\right), \mathrm{R}_{2}\left(\mathrm{~T}_{2}\right)$ and steady-state NOE spectra were processed so as to achieve maximum peak heights [36]. Intensities (or peak heights; in arbitrary units) for various $\left({ }^{1} \mathrm{H}_{-}{ }^{15} \mathrm{~N}\right)$ cross peaks in these spectra were measured using the Felix software. Uncertainty in the peak heights was measured from the duplicate spectra. The standard deviations were found to be $<5 \%$ for all the relaxation data. After calculating the peak heights and their respective errors, the above time points were fitted to a single exponential decay function, to derive the corresponding $\mathrm{R}_{1}$ and $\mathrm{R}_{2}$ values, where $I(t)$ is the intensity at delay $t(\mathrm{~ms})$ used in the measurement of $R_{1}$ and $R_{2}$. $A+B$ is the intensity at initial time $\mathrm{t}=0$, and $\mathrm{A}$ is the steady-state value that is the intensity at $\mathrm{t}=\infty$.

$$
\mathrm{I}(\mathrm{t})=\mathrm{A}+\mathrm{B} \exp \left(-\mathrm{R}_{1,2} \mathrm{t}\right)
$$

The ${ }^{1} \mathrm{H}-{ }^{15} \mathrm{~N}$ heteronuclear NOE was calculated from the equation,

$$
\mathrm{NOE}=\mathrm{I}_{\mathrm{sat}} / \mathrm{I}_{\mathrm{eq}}
$$

where $I_{\text {sat }}$ and $I_{\text {eq }}$ are the intensities of a peak in the spectra recorded with and without proton saturation.

\section{Reduced Spectral Density Calculation}

In the present study, we preferred the use of reduced spectral density calculation method instead of model-free analysis [37, 38]. In principle, both the methods are very robust and give similar kind of information. Wagner et al., $[25,39,40]$ described the calculation of spectral density functions by the use of $\operatorname{six}{ }^{1} \mathrm{H}$ and ${ }^{15} \mathrm{~N}$ relaxation rates. The analysis is neither independent of any form of time dependence of the autocorrelation function, nor does it require any specific kind of rotational diffusion tensor calculation of the molecule. Recent papers [21, 23, 41-44] use only three ${ }^{15} \mathrm{~N}$ relaxation parameters for reduced spectral density mapping, and thus provide a very useful method to obtain the protein motional information with the assumption that at high frequencies, the spectral density functions are: $J\left(\omega_{H}\right) \approx J\left(\omega_{H}+\omega_{N}\right) \approx J\left(\omega_{H}-\omega_{N}\right)$. Using the method described by Lefevre et al., we calculated $J(0), J\left(\omega_{N}\right)$, and $J\left(\omega_{H}\right)$, and then examined the linear correlation between $J(0)$ and $J\left(\omega_{N}\right)$, and $J(0)$ and $J\left(\omega_{H}\right)$. Using same approach, $J(0), J\left(\omega_{N}\right)$, and $J\left(\omega_{H}\right)$ can be written in terms of ${ }^{15} \mathrm{~N}$ longitudinal (R1) and transverse (R2) relaxation rates and heteronuclear $\left[{ }^{1} \mathrm{H}-{ }^{15} \mathrm{~N}\right]$ NOEs.

$$
\begin{aligned}
& J(0)=\frac{3}{2\left(3 d^{2}+c^{2}\right)}\left[-\frac{1}{2} R_{1}+R_{2}-\frac{3}{5} R_{N O E}\right] \\
& J\left(\omega_{N}\right)=\frac{1}{3 d^{2}+c^{2}}\left[R_{1}-\frac{7}{5} R_{N O E}\right] \\
& J\left(\omega_{H}\right)=\frac{1}{5 d^{2}} R_{N O E}
\end{aligned}
$$

where,

$$
R_{N O E}=\left[\left(\left\{{ }^{1} H\right\}-{ }^{15} N\right) N O E-1\right] R_{1} \frac{\gamma_{N}}{\gamma_{H}}
$$

The constant $\mathrm{d}^{2}$ is approximately equal to $1.35 \times 10^{9}$ $(\mathrm{rad} / \mathrm{s})^{2}$ at both 500 and $600 \mathrm{MHz}$, whereas the constant $\mathrm{c}^{2}$ is approximately $0.87 \times 10^{9}(\mathrm{rad} / \mathrm{s})^{2}$ and $1.25 \times 10^{9}(\mathrm{rad} / \mathrm{s})^{2}$ at 500 and $600 \mathrm{MHz}$, respectively. Errors for the spectral density functions were calculated from the error in the relation parameters and by solving the above equations.

\section{ACKNOWLEDGEMENTS}

We gratefully acknowledge the National Facility for High Field NMR, supported by Department of Science and Technology (DST), Department of Biotechnology (DBT), Council of Scientific and Industrial Research (CSIR) and Tata Institute of Fundamental Research (TIFR), Mumbai. We also acknowledge Dr. S. Nanduri for initialising this project and Dr. Jun Qin from Cleveland for providing the dsRBD clone. We also thank Dhanashree Dhananjay Jagtap from NIRRH for reading the manuscript.

\section{REFERENCES}

[1] Bass BL, Hurst SR, Singer JD. Binding properties of newly identified Xenopus proteins containing dsRNA-binding motifs. Curr Biol 1994; 4: 301-14.

[2] St JD, Brown NH, Gall JG, Jantsch M. A conserved doublestranded RNA-binding domain. Proc Natl Acad Sci USA 1992; 89: 10979-83.

[3] Krovat BC, Jantsch MF. Comparative mutational analysis of the double-stranded RNA binding domains of Xenopus laevis RNAbinding protein A. J Biol Chem1996; 271: 28112-19.

[4] Schmedt C, Green SR, Manche L, Taylor DR, Ma Y, Mathews MB. Functional characterization of the RNA-binding domain and motif of the double-stranded RNA-dependent protein kinase DAI (PKR). J Mol Biol 1995; 249: 29-44.

[5] Doyle M, Jantsch MF. New and old roles of the double-stranded RNA-binding domain. J Struct Biol 2002; 140: 147-53.

[6] Gelev V, Aktas H, Marintchev A, et al. Mapping of the autoinhibitory interactions of protein kinase $\mathrm{R}$ by nuclear magnetic resonance. J Mol Biol 2006; 364: 352-63.

[7] Li S, Peters GA, Ding K, Zhang X, Qin J, Sen GC. Molecular basis for PKR activation by PACT or dsRNA. Proc Natl Acad Sci USA 2006; 103: 10005-10.

[8] Shimazawa M, Hara H. Inhibitor of double stranded RNAdependent protein kinase protects against cell damage induced by ER stress. Neurosci Lett 2006; 409: 192-5.

[9] Green SR, Mathews MB. Two RNA-binding motifs in the doublestranded RNA-activated protein kinase, DAI. Genes Dev 1992; 6 : 2478-90.

[10] McCormack SJ, Ortega LG, Doohan JP, Samuel CE. Mechanism of interferon action motif I of the interferon-induced, RNA-dependent protein kinase (PKR) is sufficient to mediate RNA-binding activity.Virology 1994; 198: 92-9.

[11] Romano PR, Green SR, Barber GN, Mathews MB, Hinnebusch AG. Structural requirements for double-stranded RNA binding, dimerization, and activation of the human eIF-2 alpha kinase DAI in Saccharomyces cerevisiae. Mol Cell Biol 1995; 15: 365-78.

[12] Nanduri S, Carpick BW, Yang Y, Williams BR, Qin J. Structure of the double-stranded RNA-binding domain of the protein kinase PKR reveals the molecular basis of its dsRNA-mediated activation. EMBO J 1998; 17: 5458-65.

[13] Barnwal RP, Chaudhuri TR, Nanduri S, Qin J, Chary KV. Methyl dynamics for understanding hydrophobic core packing of dynamically different motifs of double-stranded RNA binding domain of protein kinase R. Proteins 2006; 62: 501-08.

[14] Nanduri S, Rahman F, Williams BR, Qin J. A dynamically tuned double-stranded RNA binding mechanism for the activation of antiviral kinase PKR. EMBO J 2000; 19: 5567-74.

[15] Hansen MR, Hanson P, Pardi A. Filamentous bacteriophage for aligning RNA, DNA, and proteins for measurement of nuclear magnetic resonance dipolar coupling interactions. Methods Enzymol 2000; 317: 220-40. 
[16] Prestegard JH, Al-Hashimi HM, Tolman JR. NMR structures of biomolecules using field oriented media and residual dipolar couplings. Q Rev Biophys 2000; 33: 371-424.

[17] Tsuboi M, Kubo Y, Ikeda T, Overman SA, Osman O, Thomas GJ, Jr. Protein and DNA residue orientations in the filamentous virus Pf1 determined by polarized Raman and polarized FTIR spectroscopy. Biochemistry 2003; 42: 940-50.

[18] Kishchenko G, Makowski L. Shuffling of structural elements in filamentous bacteriophages. Proteins 1997; 27: 405-9.

[19] Thiriot DS, Nevzorov AA, Opella SJ Structural basis of the temperature transition of Pf1 bacteriophage. Protein Sci 2005; 14:1064-70

[20] Makowski L. Structural constraints on the display of foreign peptides on filamentous bacteriophages. Gene 1993; 128: 5-11.

[21] Kay LE, Torchia DA, Bax A. Backbone dynamics of proteins as studied by $15 \mathrm{~N}$ inverse detected heteronuclear NMR spectroscopy: application to staphylococcal nuclease. Biochemistry 1989; 28: 8972-9.

[22] Tjandra N, Garrett DS, Gronenborn AM, Bax A, Clore GM. Defining long range order in NMR structure determination from the dependence of heteronuclear relaxation times on rotational diffusion anisotropy. Nat Struct Biol 1997; 4: 443-9.

[23] Lefevre JF, Dayie KT, Peng JW, Wagner G. Internal mobility in the partially folded DNA binding and dimerization domains of GAL4: NMR analysis of the N-H spectral density functions. Biochemistry 1996; 35: 2674-86.

[24] Zhang P, Dayie KT, Wagner G. Unusual lack of internal mobility and fast overall tumbling in oxidized flavodoxin from Anacystis nidulans. J Mol Biol 1997; 227: 443-55.

[25] Peng JW, Wagner G. Frequency spectrum of NH bonds in eglin c from spectral density mapping at multiple fields. Biochemistry 1995; 34: 16733-52.

[26] Ramos A, Grunert S, Adams J, et al. RNA recognition by a Staufen double-stranded RNA-binding domain. EMBO J 2000; 19: 9971009.

[27] Ryter JM, Schultz SC. Molecular basis of double-stranded RNAprotein interactions: structure of a dsRNA-binding domain complexed with dsRNA. EMBO J 1998; 17: 7505-13.

[28] Wu H, Henras A, Chanfreau G, Feigon J. Structural basis for recognition of the AGNN tetraloop RNA fold by the doublestranded RNA-binding domain of Rnt1p RNase III. Proc Natl Acad Sci USA 2004; 101: 8307-12.

[29] Allain FH, Gilbert DE, Bouvet P, Feigon J. Solution structure of the two N-terminal RNA-binding domains of nucleolin and NMR study of the interaction with its RNA target. J Mol Biol 2000; 303: $227-41$.

[30] Handa N, Nureki O, Kurimoto $\mathrm{K}$, et al. Structural basis for recognition of the tra mRNA precursor by the Sex-lethal protein. Nature 1999; 398: 579-85.
[31] Nanduri S, Carpick B, Yang Y, Williams BR, Qin J. 1H, 13C, 15N resonance assignment of the $20 \mathrm{kDa}$ double stranded RNA binding domain of PKR. J Biomol NMR 1998; 12: 349-51.

[32] Barnwal RP, Atreya HS, Chary KV. Chemical shift based editing of $\mathrm{CH} 3$ groups in fractionally 13C-labelled proteins using GFT (3, 2)D CT-HCCH-COSY: stereospecific assignments of $\mathrm{CH} 3$ groups of Val and Leu residues. J Biomol NMR 2008; 42(2): 149-54.

[33] Barnwal RP, Rout AK, Atreya HS, Chary KV. Identification of Cterminal neighbours of amino acid residues without an aliphatic 13Cgamma as an aid to NMR assignments in proteins. J Biomol NMR 2008; 41(4): 191-7.

[34] Barnwal RP, Rout AK, Chary KV, Atreya HS. Rapid measurement of $3 \mathrm{~J}(\mathrm{H} \mathrm{N}-\mathrm{H}$ alpha) and $3 \mathrm{~J}(\mathrm{~N}-\mathrm{H}$ beta) coupling constants in polypeptides. J Biomol NMR 2007; 39(4): 259-63.

[35] Farrow NA, Muhandiram R, Singer AU, et al. Backbone dynamics of a free and phosphopeptide-complexed Src homology 2 domain studied by $15 \mathrm{~N}$ NMR relaxation. Biochemistry 1994; 33: 59846003.

[36] Skelton NJ, Palmer AG, Akke M, Kordel J, Rance M, Chazin WJ Practical aspects of two-dimensional proteon-detected $15 \mathrm{~N}$ spin relaxation measurements. J Magn Reson B 1993; 102: 253-64.

[37] Mandel AM, Akke M, Palmer AG, III. Backbone dynamics of Escherichia coli ribonuclease HI: correlations with structure and function in an active enzyme. J Mol Biol 1995; 246: 144-63.

[38] Mandel AM, Akke M, Palmer AG, III. Dynamics of ribonuclease $\mathrm{H}$ : temperature dependence of motions on multiple time scales. Biochemistry 1996; 35: 16009-23.

[39] Peng JW, Wagner G. Mapping of the spectral densities of N-H bond motions in eglin $\mathrm{c}$ using heteronuclear relaxation experiments. Biochemistry 1992; 31: 8571-86.

[40] Peng JW, Wagner G. Investigation of protein motions via relaxation measurements. Methods Enzymol 1994; 239: 563-96.

[41] Farrow NA, Zhang O, Szabo A, Torchia DA, Kay LE. Spectral density function mapping using $15 \mathrm{~N}$ relaxation data exclusively. J Biomol NMR 1995; 6: 153-62.

[42] Ishima R, Yamasaki K, Saito M, Nagayama K. Spectral densities of nitrogen nuclei in Escherichia coli ribonuclease HI obtained by 15N NMR relaxation and molecular dynamics. J Biomol NMR 1995; 6: 217-20.

[43] Ishima R, Nagayama K. Protein backbone dynamics revealed by quasi spectral density function analysis of amide N-15 nuclei. Biochemistry 1995; 34: 3162-71.

[44] Ishima R, Iwahara J, Yokoyama S, Nagayama K. Gaussian Spectral-Density Function for Protein Internal Motions. J Magn Reson B 1996; 111: 281-84.

[45] Koradi R, Billeter M, Wuthrich K. MOLMOL: a program for display and analysis of macromolecular structures. J Mol Graph 1996; 14: 51-32.

This is an open access article licensed under the terms of the Creative Commons Attribution Non-Commercial License (http://creativecommons.org/ licenses/by-nc/3.0/), which permits unrestricted, non-commercial use, distribution and reproduction in any medium, provided the work is properly cited. 